\title{
Next generation genome sequencing reveals phylogenetic clades with different level of virulence among Salmonella Typhimurium clinical human isolates in Hong Kong
}

Chi Keung Cheng ${ }^{1+}$, Man Kit Cheung ${ }^{1 \dagger}$, Wenyan Nong ${ }^{1}$, Patrick Tik Wan Law ${ }^{1}$, Jing Qin ${ }^{1}$, Julia Mei-Lun Ling ${ }^{2}$, Kai Man Kam, ${ }^{3,4}$ William Man Wai Cheung ${ }^{1}$ and Hoi Shan Kwan ${ }^{1 *}$

\begin{abstract}
Background: Salmonella Typhimurium is frequently isolated from foodborne infection cases in Hong Kong, but the lack of genome sequences has hindered in-depth epidemiological and phylogenetic studies. In this study, we sought to reconstruct the phylogenetic relationship and investigate the distribution and mutation patterns of virulence determinants among local S. Typhimurium clinical isolates using their genome sequences.

Results: We obtained genome sequences of 20 S. Typhimurium clinical isolates from a local hospital cluster using a 454 GS FLX Titanium sequencing platform. Phylogenetic analysis was performed based on single nucleotide polymorphism positions of the core genome against the reference strain LT2. Antimicrobial susceptibility was determined using minimal inhibitory concentration for five antimicrobial agents and analyses of virulence determinants were performed through referencing to various databases. Through phylogenetic analysis, we revealed two distinct clades of $S$. Typhimurium isolates and three outliers in Hong Kong, which differ remarkably in antimicrobial susceptibility and presentation and mutations of virulence determinants. The local isolates were not closely related to many of the previously sequenced S. Typhimurium isolates, except LT2. As the isolates in the two clades spanned over 10 years of isolation, they probably represent endemic strains. The outliers are possibly introduced from outside of Hong Kong. The close relatedness of members in one of the clades to LT2 and the Japanese stool isolate T000240 suggests the potential reemergence of LT2 progeny in regions nearby.

Conclusions: Our study demonstrated the utility of next-generation sequencing coupled to traditional microbiological testing method in a retrospective epidemiological study involving multiple clinical isolates. The evolution of multidrug- and ciprofloxacin-resistant strains among the more virulent clade is also an increasing concern.
\end{abstract}

Keywords: Foodborne infection, Epidemiology, Phylogeny, Virulence determinants, Single nucleotide polymorphism, Antimicrobial susceptibility

\footnotetext{
*Correspondence: hoishankwan@cuhk.edu.hk

${ }^{\dagger}$ Equal contributors

'School of Life Sciences, The Chinese University of Hong Kong, Hong Kong

SAR, China

Full list of author information is available at the end of the article
}

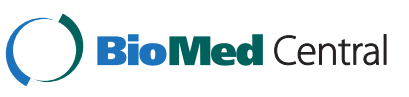

(c) 2015 Cheng et al. Open Access This article is distributed under the terms of the Creative Commons Attribution 4.0 International License (http://creativecommons.org/licenses/by/4.0/), which permits unrestricted use, distribution, and reproduction in any medium, provided you give appropriate credit to the original author(s) and the source, provide a link to the Creative Commons license, and indicate if changes were made. The Creative Commons Public Domain Dedication waiver (http://creativecommons.org/publicdomain/zero/1.0/) applies to the data made available in this article, unless otherwise stated. 


\section{Background}

Salmonella foodborne infection is a common but important public health issue worldwide. Among the many serovars, Salmonella Typhimurium is frequently isolated from outbreaks as one of the common bacterial causative agents. The World Health Organization has also emphasized the rising concern of multidrug resistance in this non-typhoid Salmonella serovar, which potentially accounts for the transfer of antimicrobial resistance to other human pathogens [1]. With the continual reduction in the cost for high-throughput genome sequencing, thousands of genomes of pathogenic bacteria have now been sequenced and Salmonella is of no exception [2]. In addition to the conventional analysis of antimicrobial resistance profiles, coupling of genome sequencing to phylogenetic analysis has opened new trends of in-depth epidemiological studies at both regional and global levels. Over the past few years, hundreds of genomes of various Salmonella serovars, including Typhimurium [3], Enteritidis [4], Typhi [5], Newport [6], Heidelberg [7], and Pullorum [8], were sequenced to facilitate evolutionary studies, as well as epidemiological and pathogenicity investigations in this important pathogen.

Despite the availability of genome sequences for $S$. Typhimurium isolates from all around the world, the Japanese strain T000240 remained as the only sequenced and published isolate from northeastern Asia [9]. Here we report the use of high-throughput genome sequencing, coupled to traditional microbiological testing method, in a retrospective study of Salmonella Typhimurium strains isolated from subjects hospitalized in Hong Kong over the past two decades. Specifically, we reconstructed the phylogenetic relationship and investigated the distribution and mutation patterns of virulence determinants among 20 local isolates.

\section{Methods}

\section{Bacterial strains}

A total of $20 S$. Typhimurium isolates (Table 1) were obtained from patients admitted to the hospitals of the New Territories East Cluster of the Hospital Authority in Hong Kong between 1993 and 2007. Written informed consent for using the blood and stool samples in the study was obtained from all participants. Seven blood isolates, three of which isolated in the mid 90's and the rest isolated in the mid 00 's, and 13 stool isolates were obtained by standard procedures. The blood isolates are representatives of the circulating clones during the sampling periods and act as representatives of systemic infection whereas the stool isolates were used as the genetic background for comparison purpose. The 10-year span between isolate collections allows determination of endemicity of the selected strains. These isolates were confirmed biochemically by the AP120E system (bioMérieux S.A., Montalieu Vercieu, France).
Table 1 Information of patients and corresponding S. Typhimurium clinical isolates

\begin{tabular}{lllll}
\hline Isolate & Source & Year of isolation & Patient age & Patient sex \\
\hline ST728/07 & Blood & 2007 & 2 & $\mathrm{~F}$ \\
ST4024/07 & Blood & 2007 & 54 & $\mathrm{M}$ \\
ST4848/06 & Blood & 2006 & 68 & $\mathrm{M}$ \\
ST2850/05 & Blood & 2005 & 81 & $\mathrm{M}$ \\
ST4650/95 & Blood & 1995 & 4 & $\mathrm{M}$ \\
ST6988/94 & Blood & 1994 & 78 & $\mathrm{~F}$ \\
ST8493/93 & Blood & 1993 & 14 & $\mathrm{~F}$ \\
ST372/06 & Stool & 2006 & 15 & $\mathrm{M}$ \\
ST1660/06 & Stool & 2006 & 8 & $\mathrm{M}$ \\
ST2286/06 & Stool & 2006 & 1 & $\mathrm{~F}$ \\
ST486/06 & Stool & 2006 & 8 & $\mathrm{~F}$ \\
ST2533/06 & Stool & 2006 & 18 & $\mathrm{M}$ \\
ST1489/06 & Stool & 2006 & 28 & $\mathrm{~F}$ \\
ST4650/06 & Stool & 2006 & 5 & $\mathrm{~F}$ \\
ST2143/05 & Stool & 2005 & 2 & $\mathrm{~F}$ \\
ST4329/05 & Stool & 2005 & 1 & $\mathrm{~F}$ \\
ST4038/02 & Stool & 2002 & 37 & $\mathrm{M}$ \\
ST3363/96 & Stool & 1996 & 7 & $\mathrm{M}$ \\
ST3858/96 & Stool & 1996 & 43 & $\mathrm{~F}$ \\
ST2287/95 & Stool & 1995 & 11 & $\mathrm{~F}$ \\
\hline
\end{tabular}

\section{Genome sequencing and de novo assembly}

Genomic DNA from the isolates was extracted using PrepMan Ultra Reagent (Applied Biosystems) according to the manufacturer's instructions. Whole-genome shotgun sequencing was performed on a 454 GS FLX Titanium platform (Roche Diagnostics) [10]. Bases sequenced and corresponding quality values were called and delivered in standard format by GS FLX for downstream bioinformatic analyses. Sequence reads were assembled de novo using Newbler assembler (Roche Diagnostics).

\section{SNPs extraction and phylogenetic analysis}

All SNP positions were obtained by aligning the genome sequences of the 20 isolates with the reference strain LT2 [11] chromosome using Mauve and 454 GS Reference Mapper [10]. Raw SNP calls were filtered to ensure that at least $90 \%$ of the reads support the SNP. SNPs called in phage sequences and repetitive regions of the reference genome were excluded. Only SNPs located in the Salmonella core genes [12] were included in the phylogenetic analysis. All remaining SNPs were concatenated to generate a single pseudo-sequence. Phylogenetic analyses were conducted in MEGA (version 5.21) [13] and phylogenetic trees were reconstructed using the Maximum Parsimony (MP) method with a heuristic search based on the Tree 
Bisection and Reconnection (TBR) approach. Salmonella Enteritidis PT4 (GenBank Accession AM933172) and Salmonella Choleraesuis SC-B67 (GenBank Accession AE017220) were used as outgroups. Nodal supports were inferred from 500 bootstrap replicates.

\section{Antimicrobials resistance profiling}

The $20 \mathrm{~S}$. Typhimurium isolates were tested for susceptibility to ampicillin, gentamicin, chloramphenicol, trimethoprim, and ciprofloxacin by an agar dilution method according to the recommendations of the Clinical and Laboratory Standards Institute (CLSI) [14]. Isolates with minimal inhibitory concentrations (MICs) greater than those for susceptible strains as suggested by CLSI were regarded as resistant. Multidrug resistance was defined as resistant to three or more of the antimicrobials tested.

\section{Virulence determinants analysis}

Genes and mutations responsible for antimicrobial resistance were retrieved from the literature and compared among the 20 isolates. Virulence factors and Salmonella Pathogenicity Islands (SPIs) for Salmonella pathogenicity were obtained from the Virulence Factors Database (VFDB) (http://www.mgc.ac.cn/VFs/) and aligned against each of the respective genome sequences for the detection of genetic variations [15]. Prophage elements for the isolates were identified by the web server PHAge Search Tool (PHAST) (http://phast.wishartlab.com/) [16].

\section{Results}

Phylogenetic tree analysis revealed two major

\section{phylogenetic clades in Hong Kong}

Genomes of 20 local $S$. Typhimurium isolates were sequenced here with an average depth of $38 \times$ (Table 2). The SNP-based phylogenetic trees grouped the $S$. Typhimurium isolates into two major phylogenetic clades (Fig. 1, Additional file 1, Additional file 2 and Additional file 3). Clade A consisted of 10 isolates with a predominance of nine stool isolates and only a single blood isolate, whereas clade B consisted of a total of seven isolates including three blood isolates and four stool isolates. The remaining three isolates appeared to be sporadic infections and they were also distantly related by themselves. Intriguingly, they were all blood isolates. The year of isolation did not seem to be an important determining factor in the phylogeny, as isolates retrieved from the 90's and 00's were both found in each of the clades.

\section{Contrasting antimicrobials resistance profiles among phylogenetic clades}

The 20 isolates were tested for their susceptibility to five antimicrobials from different classes (Table 3). Fifteen of

Table 2 Statistics for the 20 sequenced S. Typhimurium genomes

\begin{tabular}{|c|c|c|c|c|c|c|}
\hline Isolate & Total length (bp) & Read no. & Contig no. & N50 (bp) & Fold coverage & GenBank accession \\
\hline ST728/07 & $4,674,705$ & 587,022 & 51 & 297,458 & 54 & JRYT00000000 \\
\hline ST4024/07 & $4,710,407$ & 410,923 & 42 & 324,707 & 38 & JRYU00000000 \\
\hline ST4848/06 & $4,835,948$ & $1,116,351$ & 33 & 412,176 & 87 & AUXE00000000 \\
\hline ST2850/05 & $4,823,833$ & 370,563 & 49 & 324,996 & 33 & JRZV00000000 \\
\hline ST4650/95 & $4,839,422$ & 364,948 & 32 & 413,043 & 31 & $J R Z X 00000000$ \\
\hline ST6988/94 & $4,821,910$ & 570,727 & 49 & 311,185 & 47 & JRZW00000000 \\
\hline ST8493/93 & $4,821,249$ & 214,096 & 58 & 149,902 & 18 & JRZU00000000 \\
\hline ST372/06 & $4,812,346$ & 158,272 & 82 & 100,492 & 14 & JRZT00000000 \\
\hline ST1660/06 & $4,817,227$ & 689,231 & 33 & 412,269 & 55 & JRZS00000000 \\
\hline ST2286/06 & $4,680,742$ & 274,344 & 64 & 226,047 & 25 & JRZR00000000 \\
\hline ST486/06 & $4,694,852$ & 797,428 & 90 & 297,457 & 71 & JRZQ00000000 \\
\hline ST2533/06 & $4,671,245$ & 209,116 & 64 & 197,512 & 18 & JRZP00000000 \\
\hline ST1489/06 & $4,667,695$ & 240,650 & 57 & 225,759 & 18 & JRZO00000000 \\
\hline ST4650/06 & $4,667,933$ & 230,841 & 56 & 226,048 & 17 & JRZN00000000 \\
\hline ST2143/05 & $4,856,278$ & 775,034 & 37 & 458,267 & 68 & JRZM00000000 \\
\hline ST4329/05 & $4,667,023$ & 216,066 & 59 & 192,711 & 16 & JRZL00000000 \\
\hline ST4038/02 & $4,675,722$ & 301,882 & 59 & 223,187 & 25 & JRZK00000000 \\
\hline ST3363/96 & $4,675,601$ & 653,493 & 46 & 324,537 & 53 & JRZJ00000000 \\
\hline ST3858/96 & $4,672,420$ & 341,172 & 45 & 423,111 & 32 & JRZI00000000 \\
\hline ST2287/95 & $4,846,386$ & 371,971 & 36 & 412,238 & 33 & JRZH00000000 \\
\hline
\end{tabular}




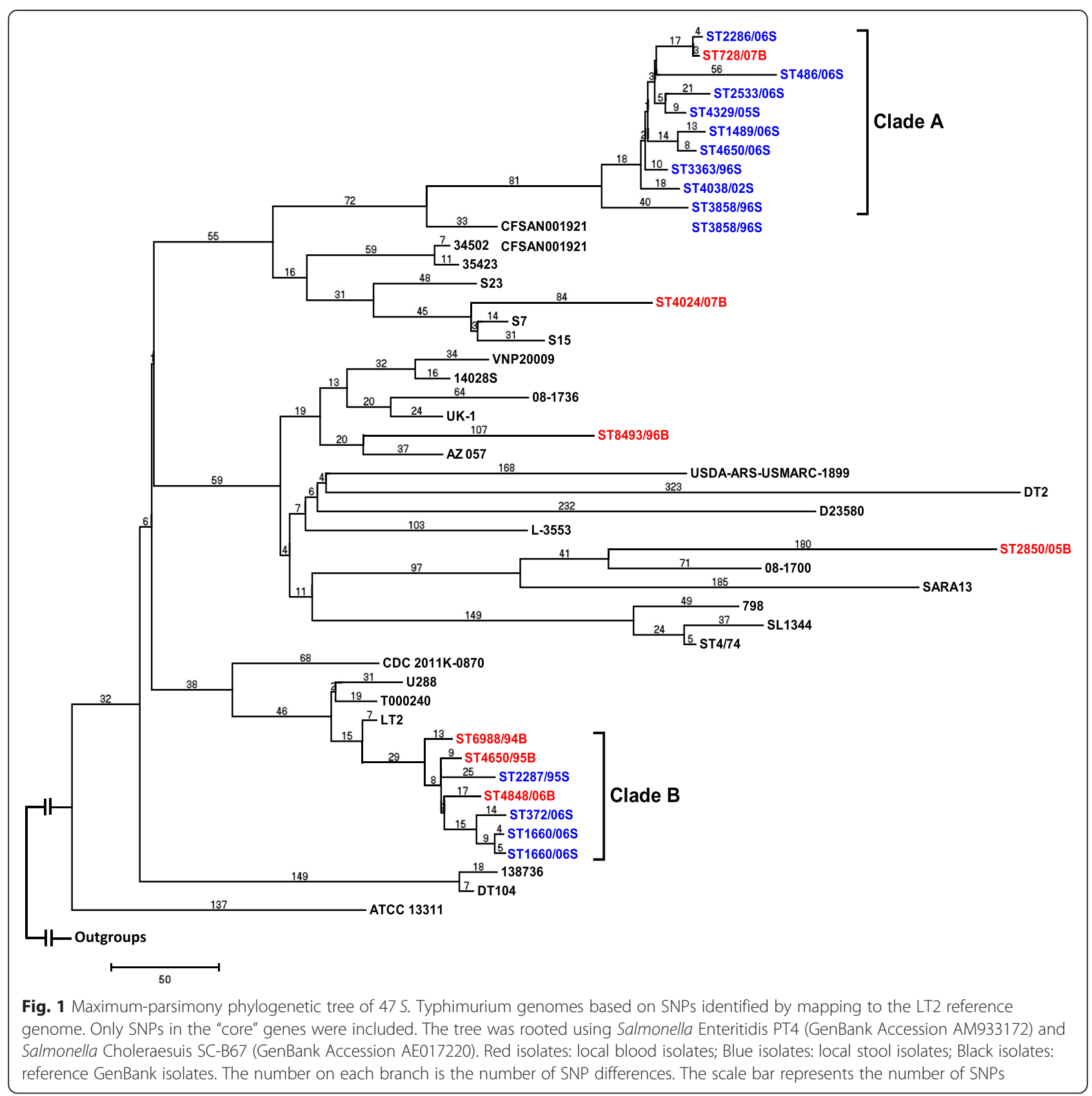

the isolates $(75 \%)$ were resistant to at least one antimicrobial class. More than half of the strains were resistant to ampicillin (70\%), trimethoprim (60\%) and chloramphenicol (55\%), while less was resistant to ciprofloxacin $(25 \%)$ and gentamicin $(20 \%)$. Except gentamicin, the proportion of resistance to ampicillin, trimethoprim, chloramphenicol, and ciprofloxacin was higher in the blood isolates (100, 86, 71 and $29 \%$ ) than the stool isolates (54, 46, 46 and $23 \%)$. Among the 20 isolates, $11(55 \%)$ were multidrug-resistant, in which five were blood isolates. Intriguingly, with respect to the phylogenetic tree above, all seven isolates within clade B were multidrug-resistant, with all of them resistant to the older antimicrobials ampicillin and chloramphenicol and six of them resistant to trimethoprim. In contrast, among the 10 isolates from clade A, only two of them were multidrug-resistant, with at least half of them still susceptible to these older antimicrobials.

\section{Loss of virulence determinants in clade $A$ isolates}

Genome sequence analysis revealed the absence of the virulence plasmid pSLT, a $\sim 90 \mathrm{~kb}$ plasmid of LT2 which 
Table 3 Susceptibility to five antimicrobials for the 20 S. Typhimurium isolates

\begin{tabular}{|c|c|c|c|c|c|c|c|c|c|}
\hline Isolate & Source & Clade & Year of isolation & AMP & GEN & $\mathrm{CHL}$ & TRI & CIP & Total \# of resistance \\
\hline ST728/07 & Blood & A & 2007 & $R$ & $S$ & $S$ & $\mathrm{R}$ & $S$ & 2 \\
\hline ST4024/07 & Blood & - & 2007 & $\mathrm{R}$ & $S$ & $\mathrm{R}$ & $\mathrm{R}$ & $\mathrm{R}$ & 4 \\
\hline ST4848/06 & Blood & B & 2006 & $\mathrm{R}$ & $\mathrm{R}$ & $\mathrm{R}$ & $\mathrm{R}$ & $\mathrm{R}$ & 5 \\
\hline ST2850/05 & Blood & - & 2005 & $\mathrm{R}$ & S & $S$ & S & S & 1 \\
\hline ST4650/95 & Blood & B & 1995 & $\mathrm{R}$ & S & $\mathrm{R}$ & $\mathrm{R}$ & S & 3 \\
\hline ST6988/94 & Blood & B & 1994 & $\mathrm{R}$ & S & $\mathrm{R}$ & $\mathrm{R}$ & S & 3 \\
\hline ST8493/93 & Blood & - & 1993 & $\mathrm{R}$ & S & $\mathrm{R}$ & $\mathrm{R}$ & S & 3 \\
\hline ST372/06 & Stool & B & 2006 & $\mathrm{R}$ & $\mathrm{R}$ & $\mathrm{R}$ & $\mathrm{R}$ & $\mathrm{R}$ & 5 \\
\hline ST1660/06 & Stool & B & 2006 & $\mathrm{R}$ & $S$ & $\mathrm{R}$ & $\mathrm{R}$ & $\mathrm{R}$ & 4 \\
\hline ST2286/06 & Stool & A & 2006 & $\mathrm{R}$ & $S$ & S & R & S & 2 \\
\hline ST486/06 & Stool & A & 2006 & $S$ & $S$ & S & S & $S$ & 0 \\
\hline ST2533/06 & Stool & A & 2006 & $\mathrm{R}$ & $\mathrm{R}$ & $S$ & R & $S$ & 3 \\
\hline ST1489/06 & Stool & A & 2006 & $S$ & $S$ & $\mathrm{R}$ & S & S & 1 \\
\hline ST4650/06 & Stool & A & 2006 & $S$ & $\mathrm{~S}$ & $S$ & $S$ & $S$ & 0 \\
\hline ST2143/05 & Stool & B & 2005 & R & S & $\mathrm{R}$ & S & $\mathrm{R}$ & 3 \\
\hline ST4329/05 & Stool & A & 2005 & S & $S$ & $S$ & S & S & 0 \\
\hline ST4038/02 & Stool & A & 2002 & S & S & $S$ & S & S & 0 \\
\hline ST3363/96 & Stool & A & 1996 & R & S & $\mathrm{R}$ & $\mathrm{R}$ & S & 3 \\
\hline ST3858/96 & Stool & A & 1996 & S & S & $S$ & S & S & 0 \\
\hline ST2287/95 & Stool & B & 1995 & $\mathrm{R}$ & $\mathrm{R}$ & R & $\mathrm{R}$ & S & 4 \\
\hline
\end{tabular}

AMP Ampicillin, GEN Gentamicin, CHL Chloramphenicol, TRI Trimethoprim, CIP Ciprofloxacin

harbors many important virulence factors including the $s p v$ locus, pef (plasmid-encoded fimbriae) locus and the complement resistance gene rck [17], in the 10 clade A isolates.

The Salmonella Pathogenicity Islands (SPIs), which encode two type III secretion systems (T3SS) and a number of virulence effectors, represent another category of important virulence factors. Genome sequences revealed that SPI1-5 were present in all 20 isolates and were highly conserved in sequence. However, a number of SNPs were found in SPIs in isolates from clade A, for instance, fhlA (nucleotide position 1916) in SPI1, orf242 (pos. 541) and sseC (pos. 1272) in SPI2 as well as sugR (pos. 183) and mgtB (pos. 351) in SPI3 (Additional file 4). The SNP in $s s e C$, which was shown to be an important effector protein to alter host cell physiology and promote bacterial survival [18], resulted in a previously undescribed Glu424 > Asp amino acid change. Another effector protein sseI/srfH, which lies within the Gifsy-2 prophage, also showed a SNP at nucleotide position 139 and resulted in a Ala47 > Thr amino acid change. Nevertheless, other effector proteins, including those encoded outside of SPI1 and SPI2 such as sopB and $\operatorname{sop} E 2$, did not show any sequence variation.
Apart from the pSLT plasmid and SNPs in the SPIs, isolates in clade A also contained less genetic materials arisen from prophages. All isolates from clade B contained a complete copy of the Salmonella prophages Gifsy-1 and Gifsy-2 [19, 20], whereas isolates from clade A contained only $\sim 39$ and $\sim 68 \%$ genetic materials from the respective prophages. This apparent reduction of genomic content had resulted in the loss of several genes previously implicated to involve in long-term systemic infection in mice (STM2585, 2586, 2596, 2597, 2635 [Gifsy-1]) and replication in macrophages (STM1031, 1033, 1041 [Gifsy-1], 2585, 2589, 2595, 2599, 2603, 2605 [Gifsy-2]) [21, 22]. While five out of the seven isolates from clade $\mathrm{B}$ contained a complete copy of Fels-2, isolates from clade A and the three sporadic isolates did not harbor this Salmonella prophage. In addition, isolates in clade $\mathrm{A}$ had also lost a total of $\sim 20 \%$ of genetic materials from phage ST104 compared to clade B isolates, whereas sporadic isolates ST2850/05 and ST8493/93 did not harbor ST104. Instead, these two isolates contained a complete copy of the phage ST64B, which is also identified in many previously sequenced Salmonella isolates but not in isolates in clade A, B, LT2 and the Japanese isolate T000240 [9]. 


\section{Discussion}

The potential reemergence of LT2 progeny

Salmonella Typhimurium is one of the most common bacterial causes of foodborne infections in Hong Kong, with 150-200 reported cases each year. Nevertheless, genomes of these clinical isolates have seldom been sequenced. In this report, we present genome sequences of $20 \mathrm{~S}$. Typhimurium clinical isolates in Hong Kong throughout 1993-2007. Phylogenetic analysis indicated that two major phylogenetic clades (represented by clade A and B in Fig. 1) had been circulating in Hong Kong for almost the past two decades, with some sporadic infections caused by phylogenetically distinct isolates. We showed that several of the previously sequenced $S$. Typhimurium isolates, including the human isolates DT104 [23] and D23580 [24], did not show high phylogenetic relatedness to isolates either in clade $\mathrm{A}$ or B (Fig. 1). Notably, isolates from clade B showed remarkable genetic relatedness to the laboratory reference strain LT2, which was originally isolated in the 1940s. Comparative genomic analysis also indicated that the Japanese isolate T000240 displays high similarity to isolates in clade B. Izumiya et al. [9] commented that multidrug-resistant progeny of LT2 might be reemerging alongside DT104 and other definitive phage-type strains, and our data suggested that such progeny of LT2 might have already reemerged in regions nearby Japan over at least the past two decades.

\section{Analysis of antimicrobial resistance determinants}

We also showed that the clade A and B isolates differed remarkably in their level of virulence in terms of antimicrobials resistance, presence of virulence plasmids and prophage elements. Not only did clade $\mathrm{B}$ comprise a higher proportion of blood isolates, all isolates within the clade were also multidrug-resistant. Genome sequence alignment revealed that none of our 20 local isolates harbor a complete copy of the Salmonella Genomic Island 1 (SGI1) found in the DT104 lineage [25]. Specifically, all isolates in clade A did not harbor any of the genomic fragments from SGI1. Intriguingly, isolates in clade B harbor an approximately $5.2 \mathrm{~kb}$-fragment originated from SGI1 (Fig. 2a), which is represented by a class 1 integron consisting of OXA-1 beta-lactamase $b l a_{\text {oxa-30, aminoglycoside resistance pro- }}$ tein $\operatorname{aadA1}$, a small multidrug resistance protein qacE $\Delta 1$ and sulfonamide resistance gene sul1. This class 1 integron is in turn located within the previously characterized $82 \mathrm{~kb}$ GI-DT12 genomic island in T000240. Together with the chloramphenicol acetyltransferase and tetracycline resistance protein tet $A$ genes [26] located 6.7 and $10.8 \mathrm{~kb}$ upstream, respectively, to the class 1 integron, this genomic island confers resistance to a number of antimicrobials including ampicillin, kanamycin, chloramphenicol, tetracycline, sulfonamide drugs, and quaternary ammonium compounds.

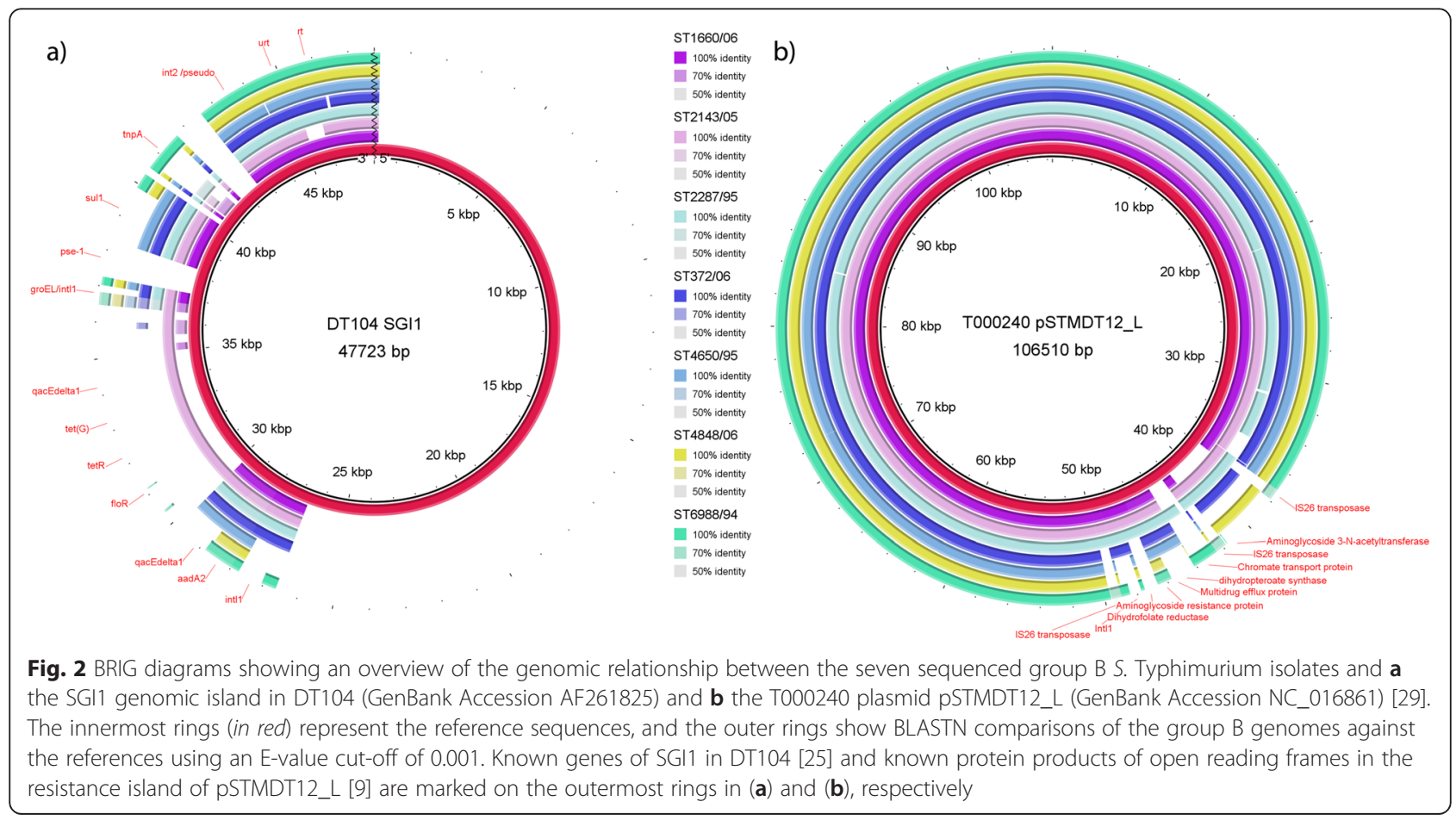


The large plasmid pSTMDT12_L identified in T000240 is also exclusively found in the clade B isolates (Fig. 2b). Nevertheless, due to the presence of four transposases (three of which are IS26) and a recombination protein in the plasmid, the resistance island showed structural variation, which results in the existence of the aminoglycoside $3-\mathrm{N}$-acetyltransferase gene (aac (3), for gentamicin resistance) in only four of the isolates and the dihydrofolate reductase gene ( $d f r A 1$, for trimethoprim resistance) in only three of the isolates.

Ciprofloxacin resistance in Salmonella Typhimurium as well as other serovars, most notably Typhi and Paratyphi, has become a global concern in recent years [27]. Five out of our 20 isolates (ST4806/06, ST372/06, ST1660/06, ST2143/05, and ST4024/07) were shown to be ciprofloxacin-resistant. The first four isolates were from clade B, and they demonstrated similar mutation patterns in the quinolone resistance-determining regions (QRDRs) in the DNA gyrase A ( gyrA) and DNA topoisomerase IV subunit $\mathrm{A}$ and $\mathrm{B}$ (parC and parE) genes [28]. Genomic sequences revealed a Ser83 $>$ Phe mutation in $g y r A$ for all the four isolates, but at amino acid 87, it was Asp87 > Asn for ST4806/06 and Asp87 > Gly for the remaining three. Mutation for parC was a consistent Ser80 > Arg, but for the parE gene it was the rarely described Leu416 > Phe for ST4806/06 and the more common Ser $458>$ Pro for the rest. No mutations were identified in the $\operatorname{gyr} B$ gene. Interestingly, the only ciprofloxacin-resistant strain outside clade B, ST4024/ 07, showed only a single mutation (Asp87 > Tyr) without any additional mutation in either $\operatorname{gyr} B, \operatorname{parC}$ or parE genes. This suggests that only a single mutation in the QRDR of gyrA is sufficient to confer resistance to ciprofloxacin.

\section{Evolution of ciprofloxacin-resistant strains}

As noted above, ciprofloxacin resistance was only identified in strains isolated in the 00's and not noted in the 90's. In particular, four of these isolates were from the more virulent clade B. Despite additional antimicrobials has not been tested, resistance to ciprofloxacin has often been associated with quinolones resistance. Such combination of multidrug and a potential quinolone resistance has prompted clinicians to pay attention to the spread of progenies from $S$. Typhimurium strains in clade B.

\section{Conclusions}

Our study revealed the existence of two major phylogenetic clades of Salmonella Typhimurium clinical isolates circulating in Hong Kong over the past two decades. The two clades differ remarkably in antimicrobial susceptibility, presentation and mutations of virulence determinants and members in one of the clades are shown to be close relatives and likely progenies of the laboratory reference strain LT2. Such potential dissemination of this multidrugresistant group of $S$. Typhimurium in the northeast Asia should deserve more attention.

\section{Availability of supporting data}

The whole genome shotgun data sets generated in this study have been deposited at DDBJ/EMBL/GenBank under the accessions JRYT00000000, JRYU00000000, AUXE00000000, JRZV00000000, JRZX00000000, JRZW 00000000, JRZU00000000, JRZT00000000, JZS00000000, JRZR00000000, JRZQ00000000, JRZP00000000, JRZO0 0000000, JRZN00000000, JRZM00000000, JRZL00000 000, JRZK00000000, JRZJ00000000, JRZI00000000, and JRZH00000000.

\section{Additional files}

\begin{abstract}
Additional file 1: Maximum-parsimony phylogenetic tree of $47 \mathrm{~S}$. Typhimurium genomes with bootstrap values reported on nodes. Only SNPs in the "core" genes were included. The tree was rooted using Salmonella Enteritidis PT4 (GenBank Accession AM933172) and Salmonella Choleraesuis SC-B67 (GenBank Accession AE017220). Red isolates: local blood isolates; Blue isolates: local stool isolates; Black isolates: reference GenBank isolates. The number at each node is the support value inferred from 500 bootstrap replicates. Bootstrap values $<50$ are not shown here. The scale bar represents the number of SNPs. (PDF $93 \mathrm{~kb}$ )

Additional file 2: Maximum-parsimony phylogenetic tree of 92 S. Typhimurium genomes based on SNPs identified by mapping to the LT2 reference genome. Only SNPs in the "core" genes were included. The tree was rooted using Salmonella Enteritidis PT4 (GenBank Accession AM933172) and Salmonella Choleraesuis SC-B67 (GenBank Accession AE017220). Red isolates: local blood isolates; Blue isolates: local stool isolates; Black isolates: reference GenBank isolates. The number on each branch is the number of SNP differences. The scale bar represents the number of SNPs. (PDF $40 \mathrm{~kb}$ )
\end{abstract}

Additional file 3: Maximum-parsimony phylogenetic tree of 92 S. Typhimurium genomes with bootstrap values reported on nodes. Only SNPS in the "core" genes were included. The tree was rooted using Salmonella Enteritidis PT4 (GenBank Accession AM933172) and Salmonella Choleraesuis SC-B67 (GenBank Accession AE017220). Red isolates: local blood isolates; Blue isolates: local stool isolates; Black isolates: reference GenBank isolates. The number at each node is the support value inferred from 500 bootstrap replicates. Bootstrap values $<50$ are not shown here. The scale bar represents the number of SNPs. (PDF $23 \mathrm{~kb}$ )

Additional file 4: SNPs located at virulence genes of $S$.

Typhimurium. The list of $S$. Typhimurium virulence genes was retrieved from VFDB. Nucleotide positions were based on the LT2 reference genome. (XLSX $54 \mathrm{~kb}$ )

Competing interests

The authors declare that they have no competing interests

Authors' contributions

CKC performed the experiments, analyzed the data and drafted the manuscript. MKC performed the phylogenetic analyses and BRIG analyses and revised the manuscript. WN performed genome assembly and SNP calling. PTWL carried out genome sequencing. JQ assisted data analysis. JMLL collected and characterized the bacterial strains. HSK conceived and designed the study. KMK and WMWC assisted in design of the study. All authors have read and approved the final manuscript. 


\section{Acknowledgements}

This work was supported by the Research Fund for the Control of Infectious Diseases (CHP-PH-06) from the Food and Health Bureau of Hong Kong SAR, China.

\begin{abstract}
Author details
'School of Life Sciences, The Chinese University of Hong Kong, Hong Kong SAR, China. ${ }^{2}$ Department of Microbiology, The Chinese University of Hong Kong, Prince of Wales Hospital, Hong Kong SAR, China. ${ }^{3}$ Centre for Health Protection, Department of Health, Hong Kong SAR, China. ${ }^{4}$ Current address: Stanley Ho Centre for Emerging Infectious Diseases, JC School of Public Health, Faculty of Medicine, The Chinese University of Hong Kong, Hong Kong SAR, China.
\end{abstract}

Received: 23 October 2014 Accepted: 8 September 2015 Published online: 14 September 2015

\section{References}

1. Weinberger $M$, Keller N. Recent trends in the epidemiology of non-typhoid Salmonella and antimicrobial resistance: the Israeli experience and worldwide review. Curr Opin Infect Dis. 2005;18:513-21.

2. Ribeiro FJ, Przybylski D, Yin S, Sharpe T, Gnerre S, Abouelleil A, et al. Finished bacterial genomes from shotgun sequence data. Genome Res. 2012;22:2270-7.

3. Luo Y, Kong Q, Yang J, Golden G, Wanda SY, Jensen RV, et al. Complete genome sequence of the universal killer Salmonella enterica Serovar Typhimurium UK-1 (ATCC 68169). J Bacteriol. 2011;193:4035-6.

4. Betancor L, Yim L, Martínez A, Fookes M, Sasias S, Schelotto F, et al. Genomic comparison of the closely related Salmonella enterica Serovars Enteritidis and Dublin. Open Microbiol J. 2012;6:5-13.

5. Baker S, Dougan G. The genome of Salmonella enterica serovar Typhi. Clin Infect Dis. 2007:45:S29-33.

6. Cao G, Zhao S, Strain E, Luo Y, Timme R, Wang C, et al. Draft genome sequences of eight Salmonella enterica serotype newport strains from diverse hosts and locations. J Bacteriol. 2012;194:5146.

7. Hoffmann M, Zhao S, Luo Y, Li C, Folster JP, Whichard J, et al. Genome sequences of five Salmonella enterica serovar Heidelberg isolates associated with a 2011 multistate outbreak in the United States. J Bacteriol. 2012;194:3274-5.

8. Feng Y, Xu HF, Li QH, Zhang SY, Wang CX, Zhu DL, et al. Complete genome sequence of Salmonella enterica serovar pullorum RKS5078. J Bacteriol. 2012;194:744.

9. Izumiya H, Sekizuka T, Nakaya H, Taguchi M, Oguchi A, Ichikawa N, et al. Whole-genome analysis of Salmonella enterica serovar Typhimurium T000240 reveals the acquisition of a genomic island involved in multidrug resistance via IS1 derivatives on the chromosome. Antimicrob Agents Chemother. 2011;55:623-30.

10. Gilles A, Meglécz E, Pech N, Ferreira S, Malausa T, Martin JF. Accuracy and quality assessment of 454 GS-FLX Titanium pyrosequencing. BMC Genomics. 2011;12:245

11. McClelland M, Sanderson KE, Spieth J, Clifton SW, Latreille P, Courtney L, et al. Complete genome sequence of Salmonella enterica serovar Typhimurium LT2. Nature. 2001;413:852-6.

12. Leekitcharoenphon P, Lukjancenko O, Friis C, Aarestrup FM, Ussery DW. Genomic variation in Salmonella enterica core genes for epidemiological typing. BMC Genomics. 2012;13:88.

13. Tamura K, Peterson D, Peterson N, Stecher G, Nei M, Kumar S. MEGA5: molecular evolutionary genetics analysis using maximum likelihood, evolutionary distance, and maximum parsimony methods. Mol Biol Evol. 2011;28:2731-9.

14. Jorgensen JH, Hindler JF. New consensus guidelines from the clinical and laboratory standards institute for antimicrobial susceptibility testing of infrequently isolated or fastidious bacteria. Clin Infect Dis. 2007:44:280-6.

15. Chen L, Xiong Z, Sun L, Yang J, Jin Q. VFDB 2012 update: toward the genetic diversity and molecular evolution of bacterial virulence factors. Nucleic Acids Res. 2012;2012(40):D641-645.

16. Zhou Y, Liang Y, Lynch KH, Dennis JJ, Wishart DS. PHAST: a fast phage search tool. Nucleic Acids Res. 2011;39:W347-352.

17. Matsui H, Bacot CM, Garlington WA, Doyle TJ, Roberts S, Gulig PA. Virulence plasmid-borne spvB and spvC genes can replace the 90-kilobase plasmid in conferring virulence to Salmonella enterica serovar Typhimurium in subcutaneously inoculated mice. J Bacteriol. 2001;183:4652-8.
18. Klein JR, Jones BD. Salmonella pathogenicity island 2-encoded proteins SseC and SseD are essential for virulence and are substrates of the type III secretion system. Infect Immun. 2001;69:737-43.

19. Stanley $T L$, Ellermeier CD, Slauch JM. Tissue-specific gene expression identifies a gene in the lysogenic phage Gifsy-1 that affects Salmonella enterica serovar typhimurium survival in Peyer's patches. J Bacteriol. 2000;182:4406-13.

20. Ho TD, Slauch JM. Characterization of grvA, an antivirulence gene on the gifsy-2 phage in Salmonella enterica serovar typhimurium. J Bacteriol. 2001;183:611-20.

21. Lawley TD, Chan K, Thompson LJ, Kim CC, Govoni GR, Monack DM. Genome-wide screen for Salmonella genes required for long-term systemic infection of the mouse. PLoS Pathog. 2006;2:e11.

22. Klumpp J, Fuchs TM. Identification of novel genes in genomic islands that contribute to Salmonella typhimurium replication in macrophages. Microbiology. 2007;153:1207-20.

23. Cooke FJ, Brown DJ, Fookes M, Pickard D, Ivens A, Wain J, et al. Characterization of the genomes of a diverse collection of Salmonella enterica serovar Typhimurium definitive phage type 104. J Bacteriol. 2008;190:8155-62.

24. Kingsley RA, Msefula CL, Thomson NR, Kariuki S, Holt KE, Gordon MA, et al. Epidemic multiple drug resistant Salmonella Typhimurium causing invasive disease in sub-Saharan Africa have a distinct genotype. Genome Res. 2009;19:2279-87.

25. Mulvey MR, Boyd DA, Olson AB, Doublet B, Cloeckaert A. The genetics of Salmonella genomic island 1. Microbes Infect. 2006:8:1915-22.

26. Cabrera R, Ruiz J, Marco F, Oliveira I, Arroyo M, Aladueña A, et al. Mechanism of resistance to several antimicrobial agents in Salmonella clinical isolates causing traveler's diarrhea. Antimicrob Agents Chemother. 2004;48:3934-9.

27. Capoor MR, Nair D, Walia NS, Routela RS, Grover SS, Deb M, et al. Molecular analysis of high-level ciprofloxacin resistance in Salmonella enterica serovar Typhi and S. Paratyphi A: need to expand the QRDR region? Epidemiol Infect. 2009;137:871-8.

28. Kehrenberg C, de Jong A, Friederichs S, Cloeckaert A, Schwarz S. Molecular mechanisms of decreased susceptibility to fluoroquinolones in avian Salmonella serovars and their mutants selected during the determination of mutant prevention concentrations. J Antimicrob Chemother. 2007:59:886-92.

29. Alikhan NF, Petty NK, Ben Zakour NL, Beatson SA. BLAST Ring Image Generator (BRIG): simple prokaryote genome comparisons. BMC Genomics. 2011;12:402

\section{Submit your next manuscript to BioMed Central and take full advantage of:}

- Convenient online submission

- Thorough peer review

- No space constraints or color figure charges

- Immediate publication on acceptance

- Inclusion in PubMed, CAS, Scopus and Google Scholar

- Research which is freely available for redistribution 